\title{
Reducing low-value interventions in the emergency department: you may be part of the problem
}

\author{
Inderjeet S. Sahota, MD, MSc*; Eddy Lang, $\mathrm{MD}^{\dagger}$ \\ STEWARDSHIP OF RESOURCES
}

The CanMEDS framework that guides medical education in Canada by determining the core competencies that all practicing physicians should have recognizes the importance of stewardship of resources. Indeed, it speaks directly to the need for physicians to allocate health resources in a judicious and cost-appropriate manner. ${ }^{1}$ Stewardship of resources is recognized as a shared responsibility for all physicians. Unfortunately, in the ordinary day of a physician, other CanMEDS roles, such as medical expert and health advocate, take precedent, and oftentimes the judicious use of health care dollars is left out of sight and out of mind. However, wasteful practices are a major problem in our emergency departments (EDs), and this problem must be addressed. This editorial aims to highlight factors at the root cause of wastage in the ED and discuss possible solutions that we as a profession could implement to tackle this growing problem.

\section{A ROLE FOR WASTE REDUCTION EDUCATION IN MEDICAL TRAINING}

Many who recall our days as students will appreciate that making ends meet on a fixed budget was a fact of life. However, as physicians and trainees, we can remain insulated from resource consumption considerations when caring for our patients, with only cursory exposure to and knowledge of the costs involved in administering the care we deliver. ${ }^{2}$ But why is that the case?

An editorial from the University of Toronto highlights how this problem begins in the earliest development of physicians. ${ }^{3}$ The authors felt that, although stewardship was an increasingly important component of medicine, teaching medical students about stewardship was lacking in traditional education. Instead, contemporary medical education celebrates thoroughness and curiosity in a bid to identify the "zebras" of clinical medicine - sometimes at the expense of increased costs to the system and increased anxiety to the patient. Another study ${ }^{4}$ on this topic also found that numerous factors, including the relative wealth of the region in which the physician trained, influence spending habits in practice. Reducing waste will require a cultural shift from one where long differential diagnoses generate extensive and unreasonable investigations to one where restraint is celebrated by keeping the focus on the most likely entities that need to be investigated. This needs to start at the undergraduate medical education level.

\section{THE RISING COSTS OF ED-UTILIZATION IN CANADA}

In 2005-2006, a Canadian Institute for Health Information report ${ }^{5}$ found that in Canada we spent $\$ 1.8$ billion (or $4 \%$ of total hospital budgets) on our EDs, although this estimate may not discern costs attributed to admitting services or consultants who see patients in the ED. A Canadian Federation for Healthcare Improvement report ${ }^{6}$ released in 2013 stated that if the current rate of ED use and spending is not kept in check, the annual number of emergency visits could increase from 15 million to 21 million, an increase of $40 \%$, over the next 30 years. This is driven by many factors, including limited access to primary care and the impact of an aging population suffering from more complex chronic disease.

Although the increase in ED visits may point to larger systems-level problems in the way that health care is delivered, such as lack of primary care or long-term

From the ${ }^{*}$ Cumming School of Medicine and tDepartment of Emergency Medicine, Cumming School of Medicine, University of Calgary, Calgary, AB.

Correspondence to: Dr. Eddy Lang, Rockyview General Hospital, 7007 - 14 St. SW, Room 5A105, Calgary, AB T2V 1P9, Canada; Email: Eddy. Lang@albertahealthservices.ca.

(C) Canadian Association of Emergency Physicians

CJEM 2017;19(2):143-146

DOI 10.1017/cem.2016.349 
assisted living for the elderly, the increased volume invariably results in increased costs, providing a greater impetus for cost-effective measures in the ED. Furthermore, there is expected to be an increase in the length of stay in Canadian EDs over the next 30 years. ${ }^{6}$ This, again, can be attributed to the country's aging population who, on average, spend more time in the ED per visit. ${ }^{6}$ ED utilization amongst the elderly is a complex topic beyond the scope of this paper. However, a recent Canadian study ${ }^{7}$ found that, although the elderly ( $>65$ years of age) accounted for only $13 \%$ of the population, they accounted for $21.8 \%$ of ED visits. Furthermore, admission rates, length of stay in hospital, need for consultation, and arrival via ambulance all increased with age. The authors argue that, although a greater number of older adults require EDs for timely care, the rapid, goal-directed model of care in the ED is not an adequate substitution for more thorough assessment and follow-up that can be provided by primary care physicians in the community.

Although some might claim that emergency medicine is a "low-tech" specialty incurring far fewer costs per patient than other areas of medicine, we should be conscious of the fact that we play a key role in triggering the admission process to the hospital, and this is often the most expensive decision made in acute care medicine. Yet, we receive little education and guidance on the topic of admission avoidance. The need to find new strategies for curbing wasteful spending in the ED is, therefore, of utmost importance.

\section{"WASTE" IN THE ED: WHAT IS IT AND WHY DOES IT EXIST?}

Wasteful interventions can be defined as those that add little or no value for patient care or are even harmful. Harm, in turn, may be defined in many ways but may include whether an unnecessary test led to further testing and treatments that were unnecessarily administered as a result of the initial test. These tests, in turn, could lead to iatrogenic physical injury or emotional distress. The dramatic growth of the Choosing Wisely ${ }^{8}$ initiative in numerous specialties and countries best reflects our growing awareness regarding the proliferation of waste in clinical medicine and the realization that the current state is unsustainable. Choosing Wisely is a campaign that aims to help clinicians and patients engage in conversations about unnecessary testing and treatments. The idea was conceived in 2010 after a paper published in the New England Fournal of
Medicine ${ }^{9}$ encouraged medical societies to create lists of five tests and treatments that were overused and did not provide meaningful benefit. The American Board of Internal Medicine Foundation further propagated this idea by providing grant funding for societies to compile these lists that eventually led to the launch of the Choosing Wisely campaign in 2012. Since then, the campaign has grown to incorporate many more medical specialties. In Canada alone, 36 medical specialty societies and physician advocacy groups have provided lists of questionable and frequently administered tests and treatments commonly seen in clinical practice. ${ }^{10}$ Indeed, the sheer number of items on these lists is a testament to the widespread prevalence of questionable interventions.

The origins of wasteful practices are not malicious in nature but have come about through a variety of trajectories. One possible trajectory is that many of these wasteful practices have simply been engrained in us or our institutions over the years, having stood the test of time but not necessarily the test of effectiveness. For example, why do we often perform coagulation testing in patients being evaluated for chest pain when they are neither anti-coagulated nor show evidence of active bleeding? Bigger ticket items like computed tomography (CT) scanning for low-risk head injury patients and CT angiography of the chest for suspected pulmonary embolism in low-risk patients are a more complex problem, and, although practice variation is well-established, ${ }^{11}$ validated clinical prediction rules can render care more rationally. Few have undertaken a systematic approach to identifying candidates for de-adoption, although this has recently been proposed by a group of Canadian critical care researchers. ${ }^{12}$ A systematic review published in 2007 examined variables affecting physicians' test-ordering tendencies. ${ }^{13}$ They found that modifiable variables that affected testordering behaviours included perceived or real medicolegal concerns, personal beliefs on the usefulness of certain tests, individual risk-taking attitudes of the physicians, and financial incentives. Other studies have corroborated these findings, ${ }^{14,15}$ as well as included additional factors, such as patient expectations and pressure from admitting services. Interestingly, awareness of the cost of tests did not influence test-ordering, providing further evidence to the idea that if a physician deems a test necessary for patient care, the cost of that test is rarely the limiting factor. ${ }^{13}$ These studies suggest that the way physicians order tests, including those we 
consider wasteful, is the product of multiple factors. Unfortunately, many of these factors, such as personal risk taking attitudes and patient expectations and pressures, may be difficult to amend system-wide. Nonetheless, studies like these do shed light on factors that may be appropriate targets for mitigating wasteful test ordering at an institutional level.

\section{MOVING FORWARD TOGETHER}

So what can the specialty do to advance the cause of high quality emergency care? Choosing Wisely is a good start with thoughtful contributions by professional organizations such as the Canadian Association of Emergency Physicians. The problem is likely greater than the items noted previously, however, and reducing waste in our EDs should not be restricted to Choosing Wisely items only. Waste reduction initiatives at the institutional level need to be driven by local considerations and local data. ${ }^{16}$ It also hinges on the buy-in and support of providers along with incentivizing local initiatives in a way that is ethical and free of any potential conflict of interest. One potential way to achieve this is a shared benefit approach. ${ }^{17}$ In this model, the costs saved by a physician or group of physicians in reducing or eliminating unnecessary testing would be re-invested into the group. For example, if eliminating unnecessary coagulation testing saves the institution " $\mathrm{x}$ " number of dollars, the department personnel that was successful in reducing these tests would be able to reinvest a proportion of those savings into a project that is meaningful to them.

Other steps that could be taken to reduce wastage include shifting the focus of diagnosis and education back into Bayesian thoughts and models instead of using "defensive medicine" principles based on "ruling out" rather than "ruling in." Emphasis on likelihood ratios of tests could provide the basis of an evidencebased framework for reasonable tests to order. Regular rounds and case discussions could be held, similar to current morbidity and mortality rounds that focus on unnecessary tests and treatments to start a discussion on value in health care and serve as reminders about what can be done at an institutional level to create change. The use of medical directives and pre-determined order sets may also reduce wastage in the ED, although the literature is less well established. A 2008 American College of Emergency Physicians report ${ }^{18}$ recommended the use of protocols, clinical prediction rules, and order sets for uniformity and in ensuring that testing and interventions occurred as soon as possible all of this in a bid to improve flow and reduce costs. Regardless of their utility, reviewing established order sets within a waste-reduction framework may be a useful strategy. As we move forward, the solution to wastage in the ED will likely require a combination of educational, administrative and, perhaps, tort reform.

Now is the time to tackle this issue. Alongside your colleagues, determine what you want on your waste reduction list and how to leverage information technology to realize these efforts. In the perfect world, items would be measureable and potentially ascribable to each physician in the ED where feedback provided in comparison with peers may provide surprising insight to providers who appreciate the existence of the problem but may not realize the extent of their contribution. Having a quality improvement lead or dedicated health researcher that can analyze and act on this list would be ideal in moving these projects forward. Other ideas include increased use of clinical decision rules and algorithms that may allow for standardized approaches to care wherein costs of diagnosis and treatment can more easily be measured and, thus, controlled. As emergency physicians, one could argue that, as a result of the limited exposure to primary care received in the Royal College residency programs, many of us are uncomfortable with discharging patients on a clinical diagnosis alone, perhaps leading to a proliferation of unnecessary tests. Increasing primary care exposure in the Royal College program, therefore, could be of use to future emergency physicians in Canada.

Ultimately, if our specialty does not aggressively address these issues, the government will eventually impose solutions upon us. The first step in transforming medical culture is increasing awareness. We must move from a culture of "what test should I order?" to one of "what will this test result change?" because as Eldridge Cleaver (an early leader in cultural change) stated, "There is no more neutrality in the world. You either have to be part of the solution, or you're going to be part of the problem."

Keywords: waste reduction, medical culture, health economics

Acknowledgements: We would like to acknowledge the reviewers of this manuscript for their helpful feedback and guidance.

Competing interests: None declared. 


\section{REFERENCES}

1. Frank JR, Snell L, Sherbino J, et al. CanMEDS 2015 physician competency framework; 2015. Available at: http://www.royalcollege.ca/portal/page/portal/rc/common/ documents/canmeds/framework/canmeds_full_framework_e. pdf (accessed 7 January 2015).

2. Innes G, Grafstein E, McGrogan J, et al. Do emergency physicians know the costs of medical care? CFEM 2000;2 (2):95-102.

3. Leon-Carlyle $M$, Srivastava R, Levinson $W$, et al. Choosing Wisely Canada: integrating stewardship in medical education. Acad Med 2015;90(11):1430.

4. Korenstein D. Charting the route to high-value care: the role of medical education. FAMA 2015;314(22):2359-61.

5. Dawson H, Zinck G. CIHI survey: ED spending in Canada: a focus on the cost of patients waiting for access to an inpatient bed in Ontario. Healthc Q 2009;12(1):25-8.

6. Canadian Foundation for Health Improvement. The impact on emergency department utilization of the CFHI Healthcare Collaborations and Initiatives; 2013. Available at: http:// www.cfhi-fcass.ca/sf-docs/default-source/reports/risk-analytica. pdf (accessed 7 January 2016).

7. Latham LP, Ackroyd-Stolarz S. Emergency department utilization by older adults: a descriptive study. Can Geriatr 7 2014;17(4):118-25.

8. Choosing Wisely Canada. Available at: http://www. choosingwiselycanada.org (accessed 7 January 2015).

9. Brody H. Medicine's ethical responsibility for health care reform - the top five list. N Engl 7 Med 2010;362(4):283-5.
10. Choosing Wisely Canada. Clinician recommendations. Available at: http://www.choosingwiselycanada.org/ recommendations/ (accessed 7 January 2015).

11. Stanley RM, Hoyle Jr. JD, Dayan PS, et al. Emergency department practice variation in computed tomography use for children with minor blunt head trauma. $\mathcal{f}$ Pediatrics 2014;165(6):1201-6.

12. Niven DJ, MCCormick TJ, Straus SE, et al. Identifying low-value clinical practices in critical care medicine: protocol for a scoping review. BM7 Open 2015;5(10):e008244.

13. Sood R, Sood A, Ghosh AK, et al. Non-evidence-based variables affecting physicians' test-ordering tendencies: a systematic review. Neth 7 Med 2007;65(5):167-77.

14. Birbeck GL, Gifford DR, Song J, et al. Do malpractice concerns, payment mechanisms, and attitudes influence testordering decisions? Neurology 2004;62(1):119-21.

15. Winkens R, Dinant GJ. Evidence base of clinical diagnosis: rational, cost effective use of investigations in clinical practice. BM7 2002;324(7340):783.

16. Bhatia RS, Levinson W, Shortt $S$, et al. Measuring the effect of Choosing Wisely: an integrated framework to assess campaign impact on low-value care. BMF Qual Saf 2015;24 (8):523-31.

17. Wilensky GR, Wolter N, Fischer MM, et al. Gain sharing: a good concept getting a bad name? Health Aff (Millwood) 2007;26(1):w58-67.

18. American College of Emergency Physicians. Emergency department crowding: high-impact solutions; 2008. Available at: http://www.acep.org/workarea/DownloadAsset. aspx?id=50026 (accessed 20 January 2015). 